\title{
A Qualitative Test for N-Finger Force-Closure Grasps on Planar Objects with Applications to Manipulation and Finger Gaits
}

\author{
I-Ming Chen and Joel W. Burdick
}

Dept. of Mechanical Engineering, Mail Code 104-44, CALTECH, Pasadena, CA 91125

\begin{abstract}
This paper presents a force-closure test function for an $n$-finger grasp on a planar object with friction. All $n$ finger grasps can be represented by an $n$-dimensional contact space. The critical conditions of the test function are used to define force-closure curves which are the boundaries of force-closure sets in the contact configuration space. We show that the force closure sets can be decomposed into subsets in which $m(m<n)$ fingers satisfy force closure. We also prove that $m=6$ is an upper bound on the order of the force closure subsets. These subsets are required for planning finger gait maneuvers which are force-closure in all phases of the gait. The characteristics of these subsets are discussed, and an algorithm to enumerate them is given. The application of the test function and the contact configuration space formulation to multifinger object manipulation and finger gait planning is demonstrated by an example.
\end{abstract}

\section{Introduction}

This paper studies the force-closure conditions for a planar object manipulated by a dextrous robot hand with $n$ fingers, or via $n$ contacts on a single robot manipulator. This problem is important to dextrous manipulation involving "finger relocation" [7] and "finger gaits" [5]. Due to finger joint limits and finger tip surface area limits, manipulation using finger tip rolling and sliding can often generate only small changes in object displacement. For large displacements, it is necessary to alternate finger relocations and roll/slide motions (such as the baton twirling example given in [4]). To reject disturbances during such manipulations, the fingers that remain in contact with the object must maintain force-closure during all roll/slide motions and gait transitions. Finger gaiting requires more than the minimum number of contacts normally required for force closure. This motivates our analysis of forceclosure constraints for a large number of fingers.

For polygonal objects, Nguyen [12] developed a test for 2-finger force-closure (FC) grasps. Both Chen and Faverjon $[1,3]$ extended Nguyen's idea to 2-finger closure on 2-D curved objects. Chen [1] also considered 2 -finger closure for smooth 3-D objects. Nevertheless, these methods cannot be generalized to 3 or more finger contacts. A quantitative test for $\mathrm{n}$-finger frictionless FC grasp of a polygon based on linear programming, has been recently proposed by Trinkle [13].

In this paper, we define a force-closure test for $n$ $(n \geq 3)$ finger contacts on a planar object with smooth boundary. This test is based on the convex hull formed by the friction cone edge wrenches produced by every contact. We term this force closure test "qualitative" because the test returns only a TRUE/FALSE value. It does not determine the optimality of a grasp configuration with respect to a given measure. We would call such a test "quantitative."

As in [1], we define an $n$-dimensional contact space, $C_{n}$, which encodes all possible finger-object contact configurations. The force-closure test is used to delineate force closure subsets of $C_{n}$. Further, we show that these sets can be decomposed into subsets in which $m(2 \leq m<n)$ fingers are force closure. These regions are required for planning finger gaiting maneuvers. Finally, we demonstrate by example how the results in this paper can be useful for planning complex multifingered manipulations which involve rolling, sliding, and finger repositioning.

\section{Preliminaries}

We assume that the grasped object boundary is a smooth and closed curve [1] which is described by: $\mathbf{p}(u)=[x(u), y(u)]^{T} . \quad u$ parametrizes distance along the object boundary. $\mathbf{p}(u)$ is a 1-to-1 function, and $\mathbf{p}\left(u_{0}\right)=\left[x\left(u_{0}\right), y\left(u_{0}\right)\right]^{T}$ represents the contact location on the object, with respect to a fixed frame $O$, at $u_{0}$. By smoothness, a unique unit tangent vector, $\mathbf{t}\left(u_{0}\right)$, and an inward pointing normal, $\mathbf{n}\left(u_{0}\right)$, exist at $u_{0}$.

Definition 2.1: The $n$-tuple $\mathbf{q}=\left(u_{1}, \ldots, u_{n}\right)$, which represents the location of $n$ point contacts on the object, with $u_{i} \neq u_{j}, i \neq j$, for $u_{i} \in \mathbf{S}^{1}, i=1, \ldots, n$, is called a contact configuration of an n-contact grasp.

Definition 2.2: Let $T^{n}=\overbrace{S^{1} \times \cdots \times S^{1}}^{n \text { times }}$ and $\Delta_{i j}=$ $\left\{\left(u_{1}, \cdots, u_{n}\right) \mid u_{i}=u_{j}, i \neq j, u_{i} \in \mathbf{S}^{1}\right\}$. The set

$$
C_{n}=\mathrm{T}^{n} \backslash\left(\bigcup_{\substack{i, j=1 \\ i \neq j, i<j}}^{n} \Delta_{i j}\right)
$$

is called the $n$-contact configuration space (or $n$-contact C-space). $\quad \Delta_{i j}$ represents all physically unrealizable contact configurations in which two contacts occupy the same location on the object. Thus, $C_{n}$ represents all possible $n$-finger grasps on the object [5].

We assume that: (1) the finger contact is point contact with friction (PCWF); (2) the contact friction is Coloumb friction, with friction coefficient $\mu$ which is everywhere constant on the object. The friction cone at the contact point becomes a sector for planar objects. 
A contact force $\mathbf{f}_{i} \in \mathbf{R}^{2}$ at $u_{i}$, along with its associated moment about the origin of $O, \tau_{i}=\mathbf{p}\left(u_{i}\right) \otimes \mathbf{f}$, is called a contact wrench $\left(\mathbf{a} \otimes \mathbf{b}=a_{1} b_{2}-a_{2} b_{1}\right.$, for $\left.\mathbf{a}, \mathbf{b} \in \mathbf{R}^{2}\right)$. It can be represented as a $3 \times 1$ vector $v_{i}=\left[\begin{array}{ll}f_{i}^{T} & \tau_{i}\end{array}\right]^{T}$. The space of all contact wrenches, termed the wrench space, is isomorphic to $\mathbf{R}^{3}$.

Let $f_{i}^{+}$and $f_{i}^{-}$be edge vectors of the friction sector at $u_{i}$ (Fig.1), where $f_{i}^{ \pm}=\mathbf{n}\left(u_{i}\right) \pm \mu t\left(u_{i}\right)$. The wrenches $w_{i}^{ \pm}=\left[\mathbf{f}_{i}^{ \pm T} \tau_{i}^{ \pm}\right]^{T}$ generated by $\mathbf{f}_{i}^{ \pm}$are termed edge wrenches $\left(\tau_{i}^{ \pm}=\mathbf{p}\left(u_{i}\right) \otimes \mathrm{f}_{i}^{ \pm}\right)$. If $\mathrm{f}_{i}$, the force exerted by the $i^{\text {th }}$ finger at $u_{i}$, lies in the friction sector, it can be expressed as positive linear combination of the edge vectors: $f_{i}=\alpha_{i}^{+} f_{i}^{+}+\alpha_{i}^{-} f_{i}^{-}$, where $\alpha_{i}^{ \pm}>0$. By linearity, a contact wrench, $v_{i}$, generated by $f_{i}$ can be expressed as the same positive linear combination of the edge wrenches: $v_{i}=\alpha_{i}^{+} w_{i}^{+}+\alpha_{i}^{-} w_{i}^{-}$. It is called a feasible contact wrench.

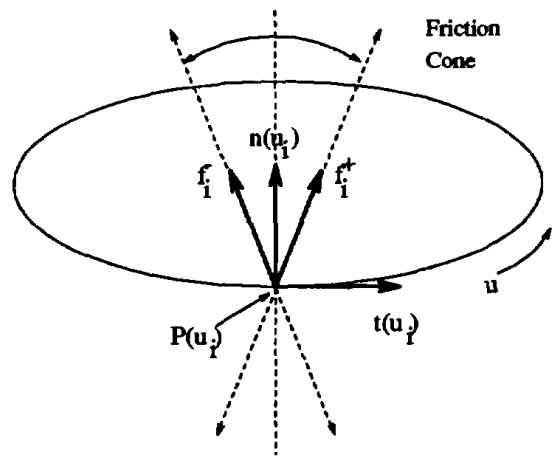

Figure 1: Friction cone and edge vectors

An n-contact force-closure grasp requires that any external wrench, $\mathbf{w}_{e}=\left[\mathbf{f}_{e}^{T} \tau_{e}\right]^{T}$, can be counter-balanced by a positive linear combination of $n$ feasible contact wrenches. Equivalently, it can be expressed as a positive linear combination of the $2 n$ edge wrenches:

$$
\begin{aligned}
\mathbf{w}_{e} & =\sum_{W \mathrm{c}}^{n} \beta_{i} \mathbf{v}_{i}=\sum^{n} a_{i}^{+} \mathbf{w}_{i}^{+}+a_{i}^{-} \mathbf{w}_{i}^{-} \\
& =W
\end{aligned}
$$

where $\beta_{i}>0, a_{i}^{ \pm}=\beta_{i} \alpha_{i}^{ \pm}>0$, and

$$
\begin{aligned}
W & =\left[\begin{array}{lllll}
w_{1}^{+} & w_{1}^{-} & \ldots & w_{n}^{+} & w_{n}^{-}
\end{array}\right], \\
c & =\left[\begin{array}{lllll}
a_{1}^{+} & a_{1}^{-} & \ldots & a_{n}^{+} & a_{n}^{-}
\end{array}\right]^{T} .
\end{aligned}
$$

$W$ is a $3 \times 2 n$ matrix termed a grasp map in [8]. Its column vectors are the contact edge wrenches. $c$ is a $2 n$-vector of edge wrench magnitudes. Note that $W$ is a function of the contact configuration: $W=W(q)$.

Let $X=\left\{x_{1}, \cdots, x_{m}\right\} \subset \mathbf{R}^{n}$ be a finite set, the convex hull of $X$, denoted by $C O(X)$, is

$$
C O(X)=\left\{\sum_{1}^{m} a_{i} x_{i} \mid a_{i} \geq 0, \sum_{1}^{m} a_{i}=1, x_{i} \in X\right\} .
$$

A hyperplane in $\mathbf{R}^{n}$ divides $\mathbf{R}^{\mathbf{n}}$ into two half spaces. A hyperplane is said to be a supporting hyperplane of $C O(X)$ if it contains a boundary point of $C O(X)$ and $C O(X)$ is contained in one of the two closed half space determined by it.

Denoting the column vectors of $W$ by $\mathbf{w}_{i}, i=1, \cdots, 2 n$, and, $C O(W) \subset \mathbf{R}^{3}$, the convex hull of the edge wrenches $\left\{w_{i}\right\}$, we have the following [8]:

Proposition 2.3: For planar object grasping with PCWF model, the following are equivalent:

1. An n-contact grasp $q$ is force-closure.

2. $\mathbf{w}_{i}$ 's of $W(\mathbf{q})$ positively span wrench space $\mathbf{R}^{3}$.

3. Let $E_{i j}$ be the plane passing through the origin of the wrench space and containing $w_{i}$ and $w_{j}$, $(i \neq j)$, for all $i, j=1, \cdots, 2 n$. None of the planes $E_{i j}$ is a supporting plane of $C O(W(\mathbf{q}))$.

4. $C O(W(\mathbf{q}))$ contains a neighborhood of the origin of the wrench space $R^{3}$.

\section{A Force-Closure Test Function}

From a computational point of view, the third statement of Proposition 2.3 gives us a plausible way to determine if $q$ is force-closure. Let $\mathbf{n}_{i j}$ be the vector normal to the plane $E_{i j}$ defined above: $\mathbf{n}_{i j}=\mathbf{w}_{i} \times \mathbf{w}_{j}$. If the inner product of $\mathbf{n}_{i j}$ with all other column vectors of $W(q)$ are of the same sign or equal to zero, $E_{i j}$ becomes a supporting plane of $C O(W)$ and $q$ is not force closure. Since each $E_{i j}$ is formed by any two of the $2 n$ column vectors of $W(q)$, there are $\left(\begin{array}{c}2 n \\ 2\end{array}\right)$ such planes which must be checked for the supporting plane condition. If none of the $E_{i j}$ is a supporting plane, $\mathrm{n}$-finger force-closure grasp is achieved.

Let SameSign $n_{k}\left[x_{1}, \cdots, x_{k}\right]$ be a True/False function of $k$ real arguments $x_{1}, \cdots, x_{k}$ such that

$$
\begin{aligned}
& \text { SameSign }\left[x_{1}, \cdots, x_{k}\right] \\
& = \begin{cases}\text { TRUE, } & \text { either } \forall i, x_{i} \geq 0, \text { or } \forall i, x_{i} \leq 0 ; \\
\text { FALSE, } & x_{i} \text { 's are not of the same sign. }\end{cases}
\end{aligned}
$$

Now define

$$
\operatorname{FACE}_{i j}(\mathbf{q})=\operatorname{SameSign}_{(2 n-2)}\left[\mathbf{n}_{i j} \cdot \mathbf{w}_{1}, \ldots, \mathbf{n}_{i j} \cdot \mathbf{w}_{m}\right],
$$

where $m=1, \cdots, 2 n, m \neq i, j$. If FACE $_{i j}=$ TRUE then $E_{i j}$ is a supporting plane.

DEFINITION 3.1: The test

$$
\text { ForceClosure }(\mathbf{q})=\neg\left(\bigvee_{\substack{i, j=1 \\ i \neq j, i<j}}^{2 n} \operatorname{FACE}_{i j}(\mathbf{q})\right)
$$

tell us the force-closure condition of an n-finger grasp q. ForceClosure $(q)=$ TRUE iff $q$ is force-closure.

Since there are $\left(\begin{array}{c}2 n \\ 2\end{array}\right)$ planes, there exists $\left(\begin{array}{c}2 n \\ 2\end{array}\right)$ FACE $_{i j}$ True/False functions in an n-finger ForceClosure test. 
The zero level set in an $a_{b}$ ument of FACE ${ }_{i j}$, i.e., the triple scalar product (TSP), $\mathbf{n}_{i j} \cdot \mathbf{w}_{k}=\left(\mathbf{w}_{i} \times \mathbf{w}_{j}\right)$. $\mathbf{w}_{k}$, defines an $(n-1)$ dimensional hyper surface in $C_{n}$ called a force-closure surface (FC-surface) and divides $C_{n}$ into two disjoint regions which have different signs of $\left(\mathbf{w}_{i} \times \mathbf{w}_{j}\right) \cdot \mathbf{w}_{k}$, so the total number of FC-surfaces in $C_{n}$ is at most $\left(\begin{array}{c}2 n \\ 2\end{array}\right) \cdot(2 n-2)$. However, by knowing the cyclic property of TSP: $(\mathbf{a} \times \mathbf{b}) \cdot \mathbf{c}=(\mathbf{b} \times \mathbf{c}) \cdot \mathbf{a}=(\mathbf{c} \times \mathbf{a}) \cdot \mathbf{b}$, for $\mathbf{a}, \mathbf{b}, \mathbf{c} \in \mathbf{R}^{3}$, the FC-surfaces $\left(\mathbf{w}_{i} \times \mathbf{w}_{j}\right) \cdot \mathbf{w}_{k}=\mathbf{0}$, $\left(\mathbf{w}_{j} \times \mathbf{w}_{k}\right) \cdot \mathbf{w}_{i}=\mathbf{0}$, and $\left(\mathbf{w}_{k} \times \mathbf{w}_{i}\right) \cdot \mathbf{w}_{j}=\mathbf{0}$ are identical. The number of FC-surfaces in $C_{n}$ is reduced to at most: $\left(\begin{array}{c}2 n \\ 3\end{array}\right)$. For 2-D objects with symmetry, e.g., a circle, this number may be further reduced.

These FC-surfaces divide the entire $\mathfrak{n}$-contact $\mathbf{C}$-space into numerous regions. The regions in which contact configurations satisfy ForceClosure(q) $=$ TRUE , are called force-closure regions (FC-regions) or feasible grasping regions [1]. While a bound on the number of FC-surfaces is known, the number of feasible grasping regions depends on the shape of the object.

EXAMPLE 3.2: Consider a two-contact grasp on an ellipse defined by $u \mapsto[4 \cos u, 2.5 \sin u], \quad 0 \leq u \leq$ $2 \pi$. $\mu=0.3$. Let $\mathbf{f}_{1}^{ \pm}, f_{2}^{ \pm}$denote the edge vectors, and the grasp map by $W=\left[\begin{array}{lllll}\mathbf{w}_{1} & \mathbf{w}_{2} & \mathbf{w}_{3} & \mathbf{w}_{4}\end{array}\right] . \mathbf{w}_{i}$ 's of $W$ will form $\left(\begin{array}{l}4 \\ 2\end{array}\right)=6$ planes $E_{i j}$, so there are $6 \mathrm{FACE}_{i j}(q)$ test functions: $\mathrm{FACE}_{12}, \mathrm{FACE}_{13}, \mathrm{FACE}_{14}, \mathrm{FACE}_{23}, \mathrm{FACE}_{24}$, FACE $_{34}$. For instance, FACE $_{12}(q)=\operatorname{SameSign}_{2}\left[\left(w_{1} \times\right.\right.$ $\left.\left.w_{2}\right) \cdot w_{3},\left(w_{1} \times w_{2}\right) \cdot w_{4}\right]$. The force-closure test is

$$
\text { ForceClosure }(\mathbf{q})=\neg\left(\bigvee_{\substack{i, j=1 \\ i \neq j, i<j}}^{4} \operatorname{FACE}_{i j}(\mathbf{q})\right)
$$

By the cyclic property of TSP, there are only 4 different FC-surfaces (FC-curves in this case):

$$
\begin{array}{ll}
\left(\mathbf{w}_{1} \times \mathbf{w}_{2}\right) \cdot \mathbf{w}_{3}(q)=0 & \left(\mathbf{w}_{1} \times \mathbf{w}_{2}\right) \cdot \mathbf{w}_{4}(q)=0 \\
\left(\mathbf{w}_{1} \times \mathbf{w}_{3}\right) \cdot \mathbf{w}_{4}(q)=0 & \left(\mathbf{w}_{2} \times \mathbf{w}_{3}\right) \cdot \mathbf{w}_{4}(q)=0
\end{array}
$$

Fig.2 illustrates the FC-curves and FC-regions in $C_{2}$ of the ellipse. The shaded regions are FC-regions. $C_{2}$, a 2-torus, can be realized in $R^{2}$ by a rectangle $I_{2 \pi} \times I_{2 \pi}$, $\left(I_{2 \pi}=[0,2 \pi]\right)$, with opposite edges identified.

\section{Symmetries of the FC-Surfaces}

FC-surfaces in $C_{n}$ have similar algebraic structures due to their symmetry with respect to permutation of the fingers. Let an n-contact grasp be $\mathbf{q}=\left(u_{1}, \cdots, u_{n}\right)$ and the grasp map be $W(\mathbf{q})$. The 3 vectors in a TSP may come from either: (1) two edge wrenches of one contact and one of the other contact, or (2) one edge wrench of each of the 3 contacts. We call (1), 2-contact FC-surfaces and (2), 3-contact FC-surfaces.

2-Contact FC-Surfaces: Four edge wrenches of 2 contacts, $u_{i}$ and $u_{j},(i, j=1, \cdots, n)$, may form 4 different TSP's, hence 4 FC-surfaces. They are

$$
\left[w_{2 i-1}\left(u_{i}\right) \times w_{2 i}\left(u_{i}\right)\right] \cdot w_{2 j-1}\left(u_{j}\right)=0
$$

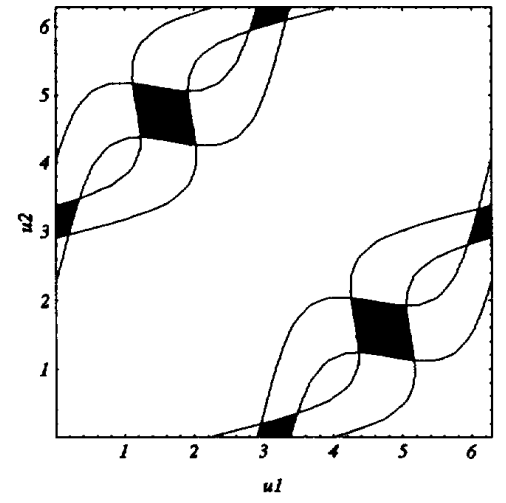

Figure 2: FC-curves and FC-regions of an ellipse

$$
\begin{aligned}
& {\left[\mathbf{w}_{2 i-1}\left(u_{i}\right) \times \mathbf{w}_{2 i}\left(u_{i}\right)\right] \cdot \mathbf{w}_{2 j}\left(u_{j}\right)=0} \\
& {\left[\mathbf{w}_{2 i-1}\left(u_{i}\right) \times \mathbf{w}_{2 j-1}\left(u_{j}\right)\right] \cdot \mathbf{w}_{2 j}\left(u_{j}\right)=0} \\
& {\left[\mathbf{w}_{2 i}\left(u_{i}\right) \times \mathbf{w}_{2 j-1}\left(u_{j}\right)\right] \cdot \mathbf{w}_{2 j}\left(u_{j}\right)=\mathbf{0}}
\end{aligned}
$$

Using the cyclic property of TSP and swapping variables: $\left\{u_{i} \rightarrow u_{j}, u_{j} \rightarrow u_{i}\right\},(4.3)$ and (4.4) become (4.1) and (4.2) respectively. There are $\left(\begin{array}{l}n \\ 2\end{array}\right)$ ways of selecting a subset of two of $n$ contacts. In total, there are $4 \cdot\left(\begin{array}{l}n \\ 2\end{array}\right)$ 2-contact FC-surfaces in $C_{n}$

3-Contact FC-Surfaces: For 3 contacts $u_{i}, u_{j}$, and $u_{k},(i, j, k=1, \cdots, n)$, the 8 edge wrenches can form 8 different TSP's, hence 8 FC-surfaces:

$$
\begin{aligned}
& {\left[w_{2 i-1}\left(u_{i}\right) \times w_{2 j-1}\left(u_{j}\right)\right] \cdot w_{2 k-1}\left(u_{k}\right)=0} \\
& {\left[w_{2 i-1}\left(u_{i}\right) \times w_{2 j-1}\left(u_{j}\right)\right] \cdot w_{2 k}\left(u_{k}\right)=0} \\
& {\left[w_{2 i-1}\left(u_{i}\right) \times w_{2 j}\left(u_{j}\right)\right] \cdot w_{2 k-1}\left(u_{k}\right)=0} \\
& {\left[w_{2 i-1}\left(u_{i}\right) \times w_{2 j}\left(u_{j}\right)\right] \cdot w_{2 k}\left(u_{k}\right)=0} \\
& {\left[w_{2 i}\left(u_{i}\right) \times w_{2 j-1}\left(u_{j}\right)\right] \cdot w_{2 k-1}\left(u_{k}\right)=0} \\
& {\left[w_{2 i}\left(u_{i}\right) \times w_{2 j-1}\left(u_{j}\right)\right] \cdot w_{2 k}\left(u_{k}\right)=0} \\
& {\left[w_{2 i}\left(u_{i}\right) \times w_{2 j}\left(u_{j}\right)\right] \cdot w_{2 k-1}\left(u_{k}\right)=0} \\
& {\left[w_{2 i}\left(u_{i}\right) \times w_{2 j}\left(u_{j}\right)\right] \cdot w_{2 k}\left(u_{k}\right)=0}
\end{aligned}
$$

Using the cyclic property and change of variables: $\left\{u_{i} \rightarrow u_{k}, u_{j} \rightarrow u_{i}, u_{k} \rightarrow u_{j}\right\}$ and $\left\{u_{i} \rightarrow u_{j}, u_{j} \rightarrow\right.$ $\left.u_{k}, u_{k} \rightarrow u_{i}\right\}$ in (4.6) and (4.8), we can obtain (4.7) and (4.11), (4.9) and (4.10) respectively. There are $\left(\begin{array}{l}n \\ 3\end{array}\right)$ ways of selecting a subset of three of $n$ contacts. Consequently, there are $8 \cdot\left(\begin{array}{l}n \\ 3\end{array}\right)$ 3-contact FC-surfaces in $C_{n}$.

Note that the total number of 2 and 3-contact FCsurfaces is: $4\left(\begin{array}{c}n \\ 2\end{array}\right)+8\left(\begin{array}{c}n \\ 3\end{array}\right)=\left(\begin{array}{c}2 n \\ 3\end{array}\right) \quad n \geq 3$. This number is equal to the number of FC-surfaces previously derived in Section 3. This implies that all FC-surfaces are either 2 or 3-contact FC-surfaces. The number of the FC-surfaces equations which must actually be computed is 6 , i.e., (4.1), (4.2), (4.5), (4.6), (4.8), and (4.12). The rest of the equations can be found by permutation of 
variables. Thus, the computation of FC-surfaces can be highly simplified.

\section{Force-Closure Contact Modes}

Assuming PCWF, at least 2 contacts are required to implement a force-closure grasp on a smooth planar object. We call such a pair of contacts an FC-2 contact. However, a 3-contact grasp can be force closure in one of two ways: (1) any two of the three contacts form force-closure or; (2) three of the contacts satisfy the closure condition, but no two of the three contacts satisfy force-closure. We call the latter type of force closure contact an $F C$ - 3 contact. In general, an $F C$ - $n$ contact is formed by $n$ contacts whose $2 n$ edge wrenches positively span the wrench space $R^{3}$ and no subset of $k(2 \leq k \leq n-1)$ contacts forms a force-closure grasp.

Definition 5.1: An n-contact grasp $q$ is called an FC$m$ contact grasp $(m \leq n)$ if $m$ of the $n$ finger contacts form a force closure grasp.

Hence, only FC-2 and FC-3 contact modes are available for 3-contact FC-grasps. But in a 4-contact FC-grasp, FC-2, FC-3, FC-4, or the combination of FC-2 and FC3 contact modes are all possible (In the last case, FC-2 and FC-3 modes share a common contact.) However, the number of FC-contact modes has an upper bound due to the following theorem from convex analysis.

Theorem: (Steinitz)[2][9] Let $X \subset \mathrm{R}^{k}$ be a finite set, i.e., $X=\left\{x_{1}, \cdots, x_{m}\right\}, x_{i} \in \mathbf{R}^{k}, i=1, \cdots, m$, and $0 \in \operatorname{Int}(C O(X))$, then there exists a $Y \subseteq X$ such that $0 \in \operatorname{Int}(C O(Y))$ with $|Y| \leq 2 k$.

Proposition 5.2: Assuming point contact with friction, there exists at most FC-6 contact modes in an $n$-contact FC-grasp on a planar object for $n \geq 6$.

Proof: From Prop.2.3, we know that in an ncontact FC-grasp q, the $2 n$ edge wrenches of $W(q)$, positively span the wrench space $\mathbf{R}^{3}$ (equiv., $0 \in$ $\operatorname{Int}(C O(W))$ ). But from Steinitz's theorem, for this case $k=3$, at most 6 of the $2 n$ edge wrenches, say $\left\{w_{a 1}, \cdots, w_{a 6}\right\}$, are needed to positively span $\mathbf{R}^{3}$, or $0 \in \operatorname{Int}\left(C O\left(\left\{w_{k 1}, \cdots, w_{k 6}\right\}\right)\right)$. Since the number of contacts $n \geq 6$, in the worst case, the 6 wrenches $\left\{w_{a 1}, \cdots, w_{a b}\right\}$ that span $R^{3}$ come from one of the two edge wrenches of each of the 6 contacts. Under this circumstance, these 6 contacts form an FC-6 contact. Any 5 or less contacts will not be force-closure. I

This proposition has the following physical interpretation. If a $n$-contact ( $n \geq 7$ ) planar grasp is forceclosure, then there must exist at least one choice of 6 contacts which are force closure. Consequently, it is always possible to lift $(n-6)$ fingers from the object surface such that the force-closure condition on the object is not disturbed. However, for a given $n$-contact ( $n \leq 6)$ force closure grasp, it may not be possible to lift a finger so that force-closure is maintained.

As stated previously, an $n$-contact FC-grasp $(n>2)$ has more than one FC contact mode, which has an advantage in planning finger gaits. Below is an algorithm to identify FC contact modes in an FC grasp. We use the set notation $\mathbf{q}_{n}=\left\{u_{1}, \cdots, u_{n}\right\}$ instead of $q$ and let $I=\{1, \cdots, n\}$ be the index set of contacts. We also use all $k$-contact $(2 \leq k \leq n-1)$ ForceClosure test functions in this algorithm and let $\mathrm{FC}_{k}\left(\mathbf{q}_{k}\right) \equiv$ ForceClosure(q), $\mathbf{q} \in C_{k}$.

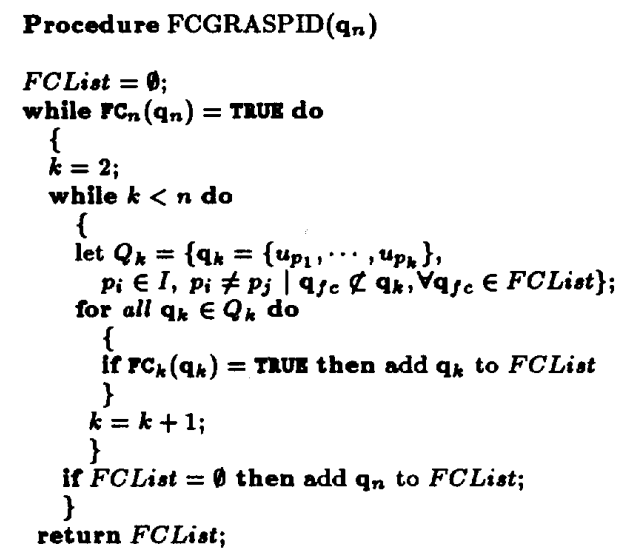

The input to FCGRASPID is $\mathbf{q}_{n}$. The output is a set FCList, which stores all contacts in $\mathrm{q}_{\mathbf{n}}$ that form FCcontacts. A member of FCList is of the form: $q_{f c}=$ $\left\{u_{p_{1}}, \cdots, u_{p_{k}}\right\}, p_{i} \in I, p_{i} \neq p_{j}, k \leq n$, which indicates that the $k$ contacts $u_{p_{1}}, \cdots, u_{p_{h}}$ in $q_{n}$ form an FC- $k$ contact. The procedure first initializes $F C L i s t$. If $\mathbf{q}_{n}$ is force-closure, then it starts to search for the FC- $k$ contacts in $q_{n}$. Line 7 generates all possible $\boldsymbol{k}$ contacts from $q_{n}$ (which are denoted by $q_{k}=\left\{u_{p_{1}}, \cdots, u_{p_{h}}\right\}$, $\left.p_{i} \in I\right)$, excludes those which contain lower FC-contact modes, and stores them in $Q_{k}$. Line 8-11 check the force-closure condition of all $\mathbf{q}_{k}$ 's in $Q_{k}$. If a $\mathbf{q}_{k}$ is force-closure, then store it in $F C L i s t$. After checking all $q_{k}$ 's, the process repeats lines 5-13 to search for FC$(k+1)$ contact modes in $q_{n}$ until $k$ is equal to $n-1$. If $F C L i s t$ is still empty after checking all FC- $(n-1), \ldots$, FC-2 contacts, $q_{n}$ is then added to $F C$ List. Line 16 returns $F C L i s t$. If $\mathbf{q}_{n}$ is not force-closure, FCList $=$ 0. If it is, FCList gives all FC-contacts in it.

\section{Characterization of the $\boldsymbol{n}$-finger FC-Sets}

We define an "FC-set" to be a set of contact configurations which satisfy the FC condition. An FC-region is a connected subset of an FC-set. The characteristics of FC-sets in $C_{2}$ have been previously studied in $[1,3]$. For grasps with more than two contacts, there exists more than one FC-contact mode. In this section, we use a 3-finger grasp example to illustrate the characteristics of higher dimensional FC-sets.

Let $\mathcal{F}_{p_{1} \ldots p_{n}}^{n},\left(p_{i} \in I\right)$, represent the FC-sets in $C_{n}$ in which the $k$ contacts $p_{1}, \cdots, p_{k}$ are FC- $k$ contact ( $k \leq n$ for $n \leq 6$, and $k \leq 6$ for $n>6$ ). For instance, all 2 -finger $\mathrm{FC}$ grasps belong to $\mathcal{F}_{12}^{2}$ in $C_{2}$. As shown in example 3.2, all shaded areas are $\mathcal{F}_{12}^{2}$. Generically, the FC-regions in $C_{3}$ are of the following types: 
(1) FC-2 contact sets: $\mathcal{F}_{12}^{3}, \mathcal{F}_{23}^{3}, \mathcal{F}_{31}^{3}$

(2) FC-3 contact sets: $\mathcal{F}_{123}^{3}$

In general, for $n \leq 6$ and $2 \leq m<n$ there will be $\left(\begin{array}{l}n \\ m\end{array}\right)$ FC-m contact sets, each arising from a given choice of $m$ fingers. For $n>6$, there are at most $\left(\begin{array}{c}n \\ 6\end{array}\right)$ FC- 6 sets.

EXAMPLE 6.1: Consider a 3-contact grasp of a circular disk described by $\theta \mapsto[r \cos \theta, r \sin \theta], 0 \leq \theta \leq 2 \pi$. $\mu=$ 0.3 . $\mathbf{q}=\left(\theta_{1}, \theta_{2}, \theta_{3}\right), \theta_{i}$ 's are contact configurations. $C_{3}=\mathrm{T}^{3} \backslash\left(\Delta_{12} \cup \Delta_{23} \cup \Delta_{31}\right)$ can be realized by a cube $I_{2 \pi} \times I_{2 \pi} \times I_{2 \pi} \in R^{3}$, with their opposite faces identified. The grasp map $W$ can be derived from (2.3).

Owing to the circular symmetry of the disk, there are only 6 FC-surfaces in $C_{3}$ :

$$
\begin{aligned}
& \theta_{1}-\theta_{2}=2 \tan ^{-1}( \pm 1 / \mu) \\
& \theta_{2}-\theta_{3}=2 \tan ^{-1}( \pm 1 / \mu) \\
& \theta_{3}-\theta_{1}=2 \tan ^{-1}( \pm 1 / \mu)
\end{aligned}
$$

When $C_{3}$ is identified with $I_{2 \times}^{3}$, those FC-surfaces become planes in $I_{2 \pi}^{3}$ as shown in Fig.3. Fig.4 shows slices of $C_{3}$ for fixed values of $\theta_{3}$. Strips (1), (2), (3) belong to FC-2 sets $\mathcal{F}_{12}^{3}, \mathcal{F}_{13}^{3}, \mathcal{F}_{23}^{3}$ respectively. Regions (4) and (5) belong to FC-3 sets $\mathcal{F}_{123}^{3}$.

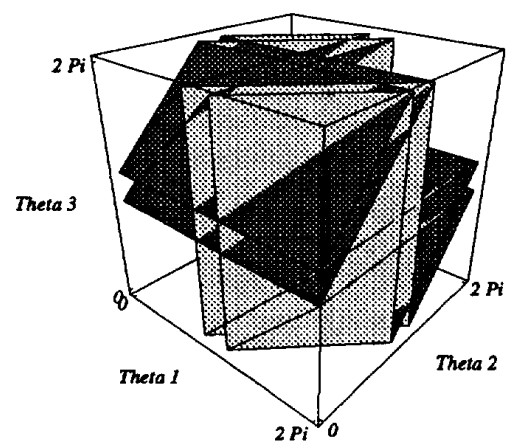

Figure 3: FC-surfaces for the disk example
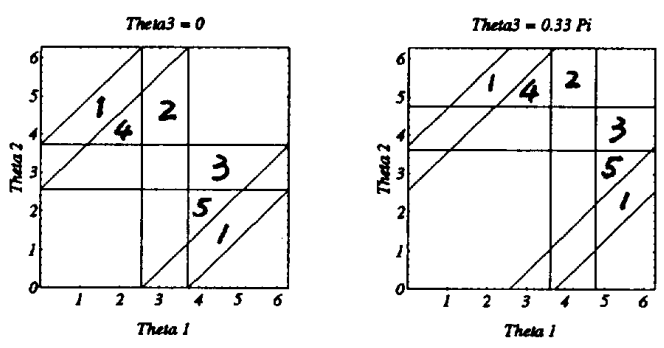

Figure 4: Constant $\theta_{3}$ slices of $C_{3}$
Definition 6.2: The intersection of different FC- $m$ $(m<n)$ contact sets are termed $m$-finger gait transition regions.

In example 6.1, the overlapping of two FC-2 contact sets implies that two different pairs of contacts in a 3-contact grasp are by themselves force closure. For instance, in $\mathcal{F}_{i j}^{3} \cap \mathcal{F}_{j k}^{3}$, it is possible to put down one finger (e.g., $k$ ) and subsequently lift another (e.g., $i$ ) while maintaining force closure in all states of the finger repositioning. These regions are essential to the implementation of finger gaits. By definition of FCcontact sets, $\mathcal{F}_{i j}^{3} \cap \mathcal{F}_{123}^{3}=\emptyset$.

\section{Application to Multifinger Manipulation}

This section discusses how to apply the results of the previous sections to planning complex manipulation tasks. Consider the planar multifinger system shown in Fig.5. Each finger has three revolute joints, and all fingers are assumed to be identical. The $i^{\text {th }}$ fingertip surface is represented by a curve $u_{f i}: I_{i} \rightarrow \mathbf{R}^{2}$ with respect to a frame, $F_{i}$, attached to the $i^{\text {th }}$ finger. As before, we assume that the grasped object is described by a curve, $\mathbf{p}(u)$. This set of fingers can reposition the grasped object using rolling, sliding, finger repositioning, or any combination of these.

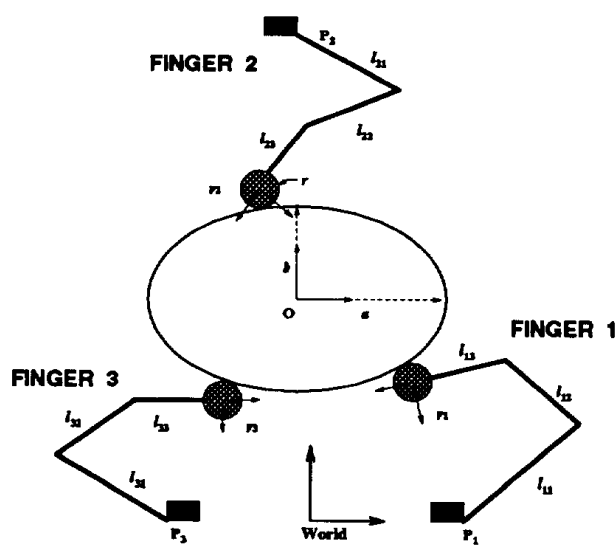

Figure 5: A planar 3-finger system

\subsection{Multifinger Manipulation}

In manipulating process, the contact locations are moving both on the finger and object surfaces. The evolution of contact points on the finger and the object during the relative motion of these objects is governed by the contact equations $[6,10,11]$, which is a set of ordinary differential equations of the contact variables defined on the object and fingers. Let $u_{o i}$ be the contact location on the object by $i^{t h}$ finger and $u_{f i}$ the corresponding contact location on the $i^{\text {th }}$ fingertip. Then $q(t)=\left(u_{o 1}(t), u_{o 2}(t), u_{o 3}(t)\right)$, the contact configuration, and $W(\mathbf{q}(t))$, the grasp map, are no longer fixed during a roll/slide manipulation. The evolution 
of $q(t)$ traces out a connected curve segment in $C_{n}$ during a continuous roll/slide sequence. To accommodate disturbance forces which arise during the execution of manipulation, force-closure must be maintained at all points along the trajectory, i.e., $\mathbf{q}(t)$ must lie in an FCregion in the contact $\mathrm{C}$-space.

In a real time situation, this can be accomplished by setting up the ForceClosure test function and checking at every instance $t$ to see if ForceClosure $(\mathbf{q}(t))=$ TRUE. The ForceClosure test depends on the object geometry and friction coefficient. If these are known in advance, then for a particular object this test function need to be set up only once.

\subsection{Finger Gaits}

Large object displacements can often not be generated purely by roll/slide manipulations because of finger joint limits, finger surface area limits, and interference between the fingers or fingers and object. Thus, finger repositioning, or "finger gaiting" will be required in addition to roll/slide motions. Finger gaits are employed to lift those fingers that have reached their joint angle or surface area limits. By readjusting the finger posture or contact location, the next phase of manipulation can be continued. A gait usually involves relocating at least two different fingers in sequence, hence, the grasp gaits must incorporate at least two different FC-contact modes. Only grasps in the gait transition regions have more than one FC-contact mode. Thus, complex object manipulations which include finger gaits must pass through a transitory state in a gait transition region.

\subsection{Dextrous Manipulation Example}

Consider using the system in fig. 5 to rotate the ellipse of example 3.2 by 120 degrees relative to its initial orientation. All fingertips are assumed to be circles of radius $r=0.7$ and there are no joint limits on the finger joints. All links can rotate 360 degree relative to their neighboring links. However, we do wish to avoid interference between the fingers and the object during the manipulation. This particular task can be accomplished by a sequence of 5 finger rolling and 4 finger gaiting motions. Fig.8 shows snapshots from a computer simulation of this complex manipulation employing the contact equations and the FC-test algorithms of the previous sections. Initially, finger 1 and 2 form an FC-2 contact in frame 1 . Frames 3 and 6 show the first rolling stage, in which finger 1,2 remain $F C-2$ contact. The rolling motion is stopped at frame 6 because of the impending interference of finger 1 and 2 with the object. The grasp is now in $\mathcal{F}_{12}^{3}$. To reposition finger 1 , finger 3 is repositioned in the grasp transition region $\mathcal{F}_{12}^{3} \cap \mathcal{F}_{23}^{3}$ so that finger 2 and 3 also form force-closure in frame 7. After adjusting the posture of finger 1 and putting it in $\mathcal{F}_{12}^{3} \cap \mathcal{F}_{23}^{3}$ (frame 8), finger 3 is relocated in $\mathcal{F}_{12}^{3} \cap \mathcal{F}_{13}^{3}$ to permit release of finger 2 (frame 9 ). Finger 2 is then readjusted and put in $\mathcal{F}_{12}^{3} \cap \mathcal{F}_{13}^{3}$ (frame 10). Again we relocate finger 3 to an appropriate place in $\mathcal{F}_{12}^{3}$ to start manipulating the object in frame 11 . By alternating the manipulating and gaiting sequence, we can obtain the desired change in object orientation shown in frame 40. In this example, all grasps are located in FC-2 contact regions so that one finger can be lifted and put down in another location. Fig. 6 shows part of the FC-2 contact regions and fig.7 shows separately the evolution of the contact points in $C_{3}$ of the ellipse. Note that the available FC grasps in the gait transition regions are restricted by the interference between fingertips. In our example, the placement of fingertips in a finger gait is chosen manually.

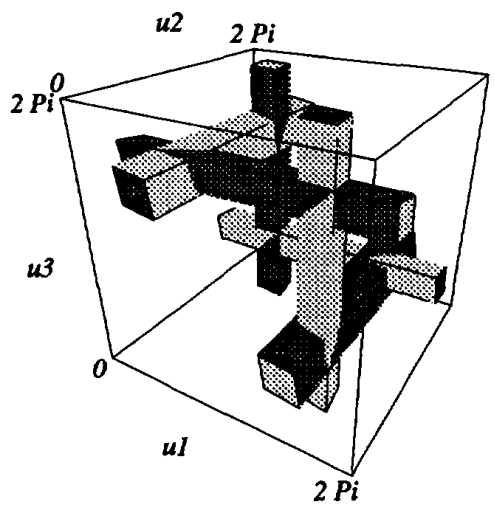

Figure 6: FC-2 contact regions in $C_{3}$ of the ellipse

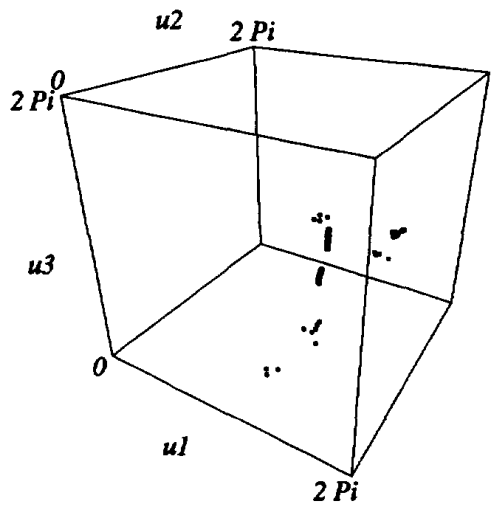

Figure 7: The trajectories of the contact points in $C_{3}$

\subsection{Manipulation with Sliding}

The above manipulation relied only on rolling motion. Sliding manipulation can be difficult to implement in practice, as it requires explicit knowledge of the contact friction coefficient. Also, the friction between two objects becomes dynamic friction during sliding, violating our static friction assumption. Nevertheless, the methods outlined in this paper can also be useful for planning sliding motion. One could plan a robust sliding motion in an FC-2 contact region so that the two non-sliding finger contacts form closure. The force and moment caused by sliding motion can be treated like disturbance on the object and can be balanced by finger contacts that form force-closure. 
FRAME 1

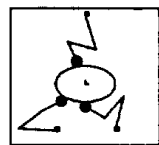

ROLLING 1

FRAME 9

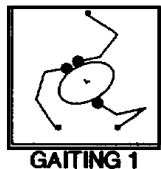

FPAME 20

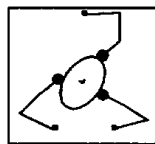

ROLIING 3
FRAME 3

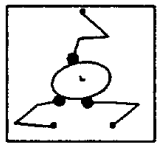

ROLING 1

FRAME 10

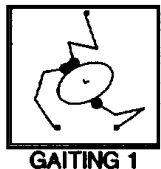

FRAME 23

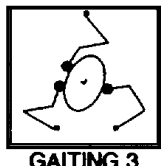

FRAME 6

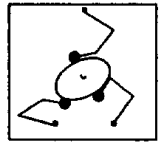

AOLING 1

FRAME 11

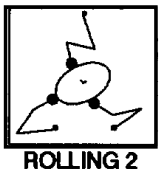

FRAME 29

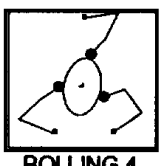

FRAME 7

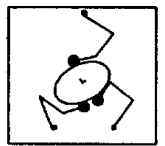

GAITING 1

FRAME 15

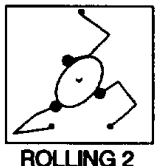

FRAME 31

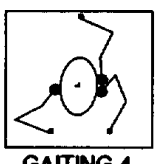

FRAME 8

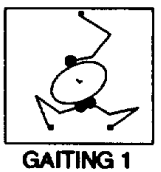

FRAME 16

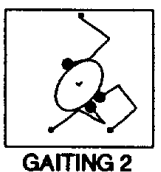

FRAME 40

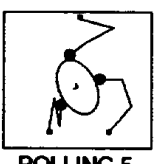

Figure 8: Snapshots of a dextrous manipulation motion sequence

\section{Summary}

We have presented here a generalized force-closure test for n-finger grasps on 2-D objects. This test is based on the convex hull of the wrenches generated by the point contact friction cone edge vectors. The critical conditions of this test function were used to define and enumerate the force closure surfaces in an ndimensional contact configuration space. These surfaces enclose regions containing contact configurations which are force closure. We showed that the force closure sets decompose into regions which correspond to closure of $m$ finger subsets of the $n$ fingers. Certain of these sub-regions, termed gait transition regions, are essential to the implementation of finger gaiting. An algorithm was presented to find these $m$ finger closure subsets. The general methodology is an useful tool for planning roll/slide motions as well. This was demonstrated by a computer simulation.

Extending this method to 3-dimensional grasping is a challenging problem. 3-D friction cones cannot be expressed as a sum of a finite set of vectors. Therefore, the convex hull condition of a finite set of wrenches can not be extended in a trivial way. If we assume a point contact model which does not support a torque about the contact normal, the friction cone can be approximated by a polygonal cone. Hence, the methods in this paper could be extended in an approximate way to this case. We hope to consider these problems in future work.

Acknowledgments: This work and the preparation of this report was supported by a Caltech President's Fund grant and an NSF Young Investigator Award MSS-9157843. We also wish to thank Prof. Richard Murray for many helpful discussions and comments.

\section{References}

[1] I.-M Chen, J. Burdick. "Finding Antipodal Point Grasps on Irregularly Shaped Objects," Proc. IEEE Int. Conf. on Robotics and Astomation, p2278-2283, Nice, France, 1992.

[2] L. Danzer, B. Grünbaum, and V. Klee. "Helly's Theorem and Its Relatives," Converity, Proc. Symp. in Pure Mathematics, p. 101-180, Vol.7, AMS, Providence, RI, 1962.

[3] B. Faverjon, J. Ponce. "On Computing Two-Finger ForceClosure Grasps of Curved 2D Objects," IEEE Int. Conf. on Robotics and Automation, p424-429, Sacramento, CA, 1991.

[4] R. Fearing. "Simplified Grasping and Manipulation with Dextrous Robot Hands," IEEE J. Robotics and Auto., Vol. 2, No. 4, 1986.

[5] J. Hong, G. Lafferriere, B. Mishra, and X. Tan. "Fine Manipulation with Multifinger Hands," IEEE Int. Conf. on Robotics and Automation, p. 1568-1573, Cincinatti, $\mathrm{OH}, 1990$.

[6] Z. Li, J. Canny. "Motion of Two Rigid Bodies with Rolling Constraint," IEEE Trans. Robotics and Automation, Vol. 6 , No. 1, Feb., 1990.

[7] Z. Li, J. Canny, and S. Sastry. "On Motion Planning for Dextrous Manipulation, Part I: The Problem Formulation," IEEE Int. Conf. on Robotics and Automation, p775-780, 1989.

[8] R. Murray, Z. Li, and S. Sastry. A Mathematical Introduction to Robotic Manipulation, preprint, CRC Press, 1993.

[9] B. Mishra, J. Schwartz, and Sharir. "On the Existence and Synthesis of Multifinger Positive Gripe," Algorithmica, p541-558, No. 2, 1987.

[10] D. Montans. "The Kinematics of Contact and Grasp," Int. J. of Robotic Research, Vol. 7, No. 3, 1988.

[11] R. Murray, S. Sastry. "Graoping and Manipulation Using Multifingered Robot Hands," Robotics, Proceedings of Symposia in Applied Mathematics, Vol. 41, AMS, Providence, RI, 1990.

[12] V. Nguyen. "Constructing Force-Closure Grasps," Int. J. of Robotic Research, vol. 7, No. 3, p3-16, June, 1988.

[13] J. Trinkle. "A Quantitative Test for Form Closure Grasps," IEEE Int. Conf. on Intelligent Robots and Systems, p1670-1677, Raleigh, NC, 1992. 